\title{
Clinical significance of hypoalbuminemia in outcome of patients with scrub typhus
}

\author{
Chang-Seop Lee ${ }^{1 *}$, In-Suk Min², Jeong-Hwan Hwang ${ }^{3}$, Keun-Sang Kwon ${ }^{4}$, Heung-Bum Lee ${ }^{1}$
}

\begin{abstract}
Background: This study was designed to investigate the clinical significance of hypoalbuminemia as a marker of severity and mortality in patients with Scrub typhus.

Methods: The patients with scrub typhus were divided into two groups based on the serum albumin levels; Group I (serum albumin $<3.0 \mathrm{~g} / \mathrm{dL}$ ) and Group II (serum albumin $\geq 3.0 \mathrm{~g} / \mathrm{dL}$ ). The outcome of patients with hypoalbuminemia was compared with that of normoalbuminemia.

Results: Of the total 246 patients who underwent the study, 84 patients (34.1\%) were categorized as Group I and 162 patients were (65.9\%) as Group II. Group I showed significantly higher incidence of confusion (24.6\% vs. 5.3\%, $p<0.001)$, pulmonary edema (15.8\% vs. $3.2 \%, p=0.002)$, pleural effusion ( $22.8 \%$ vs. $11.1 \%, p=0.03)$, arrhythmia (12.3\% vs. $2.6 \%, p=0.008)$ and non-oliguric acute renal failure $(40.4 \%$ vs. $11.1 \%, p<0.001)$ compared to group II. Hypoalbuminemic group had a higher APACHE II score (11.37 \pm 5.0 vs. $6.94 \pm 4.2, p<0.001$ ), longer hospital stay (19.9 \pm 42.1 days vs $7.5 \pm 13.8$ days, $p=0.012$ ), and higher hospital cost compared to Group II.

Conclusions: This study showed hypoalbuminemia in scrub typhus was closely related to the frequency of various complication, longer hospital stay, consequently the higher medical cost, necessitating more efficient management of patients, including medical resources.
\end{abstract}

\section{Background}

Scrub typhus is an acute febrile illness with the characteristic findings of high fever, eschar, maculopapular rash, lymphadenopathy, headache, and myalgia [1-3]. Scrub typhus caused by infection with Orientia tsutsugamushi occurs over a wide area of Eastern Asia and the Western Pacific region [1]. Usually, the symptoms of this disease are mild and its clinical course is uneventful. However, some patients experience severe or fatal events such as acute renal failure, respiratory distress or multiorgan dysfunctions $[1,4,5]$.

Generally, hypoalbuminemia is known to be associated with complications and mortality in patients with acute infectious disease [6]. In scrub typhus, about 25\% 69.2\% of patients presented hypoalbuminemia $[7,8]$. For the mechanism of hypoalbuminemia, decreased synthesis of albumin due to hepatic dysfunction, increased catabolism of protein, albuminuria, decreased intestinal absorption

\footnotetext{
* Correspondence: Icsmd@jbnu.ac.kr
${ }^{1}$ Department of Internal Medicine, Chonbuk National University Medical

* Correspondence: Icsmd@jbnu.ac.kr
'Department of Internal Medicine, Chonbuk National University Medical School and Research Institute of Clinical Medicine, Geumam-dong, Jeonju, 561180, Republic of Korea
}

(c) 2010 Lee et al; licensee BioMed Central Ltd. This is an Open Access article distributed under the terms of the Creative Commons Attribution License (http://creativecommons.org/licenses/by/2.0), which permits unrestricted use, distribution, and reproduction in any medium, provided the original work is properly cited. leakage of serum protein due to increased capillary permeability have been postulated [7].

Despite the common occurrence of hypoalbuminemia in patients with scrub typhus, there has been limited information about the relationship between the hypoalbuminemia and the severity of disease. To confirm the significance of hypoalbuminemia on the outcome of patients with scrub typhus, the clinical records of 299 patients with Scrub typhus were reviewed retrospectively.

\section{Methods}

\section{Study population and design}

A total of 302 cases (age $\geq 18$ years) who were admitted from January, 2001 through December, 2006 at Chonbuk National University Hospital, Jeonju, South Korea, and had a history of fever with either eschar or a maculopapular skin eruption and more than 2 vague symptoms (such as headache, malaise, myalgia, coughing, nausea, and abdominal discomfort) were serologically titrated and diagnosed as scrub typhus. However, 56 cases were excluded from the study because of lost 
records $(n=5)$, or the presence of underlying diseases (liver cirrhosis, $\mathrm{n}=18$; chronic obstructive pulmonary disease, $\mathrm{n}=21$; heart failure, $\mathrm{n}=5$; chronic renal failure, $\mathrm{n}=3$; acute myelogenous leukemia, $\mathrm{n}=1$; aplastic anemia, $n=3)$. The remaining 246 patients were enrolled (Figure 1). In this study, we excluded the patients with underlying diseases because the albuminemia in these cases was considered due to the host factor, which might be associated with the poor prognosis. The outcome of patients with hypoalbuminemia was compared with that of normoalbuminemia. For the purpose of this study, patients with scrub typhus with serum albumin of $<3.0 \mathrm{~g} / \mathrm{dL}$ were defined as 'Group I', while those with serum albumin of $\geq 3.0 \mathrm{~g} / \mathrm{dL}$ were defined as 'Group II'. The albumin level was checked on the first blood samples taken upon their visit to the hospital.

\section{Diagnosis of scrub typhus}

The diagnosis of scrub typhus was made on clinical manifestations and passive hemagglutination assay (PHA) against O. tsutsugamushi. A definite case of scrub typhus was defined by an increased titer of Passive Hemagglutination Assay (PHA) against O. tsutsugamushi $(\geq 1: 80)$ in a single serum sample or by a 4 -fold or greater increase of titer in the follow-up [9-11]. PHA was performed at the NeoDIN Medical Institute in Seoul, Korea using Genedia Tsutsu PHA II test kits. Genedia Tsutsu PHA II is a test kit for the qualitative and quantitative detection of antibodies against $O$. tsutsugamushi in human serum based on the PHA. In the kit, we used sheep erythrocytes sensitized by Karp and Gilliam strains, including the Boryong strain found in Korea [12,13].

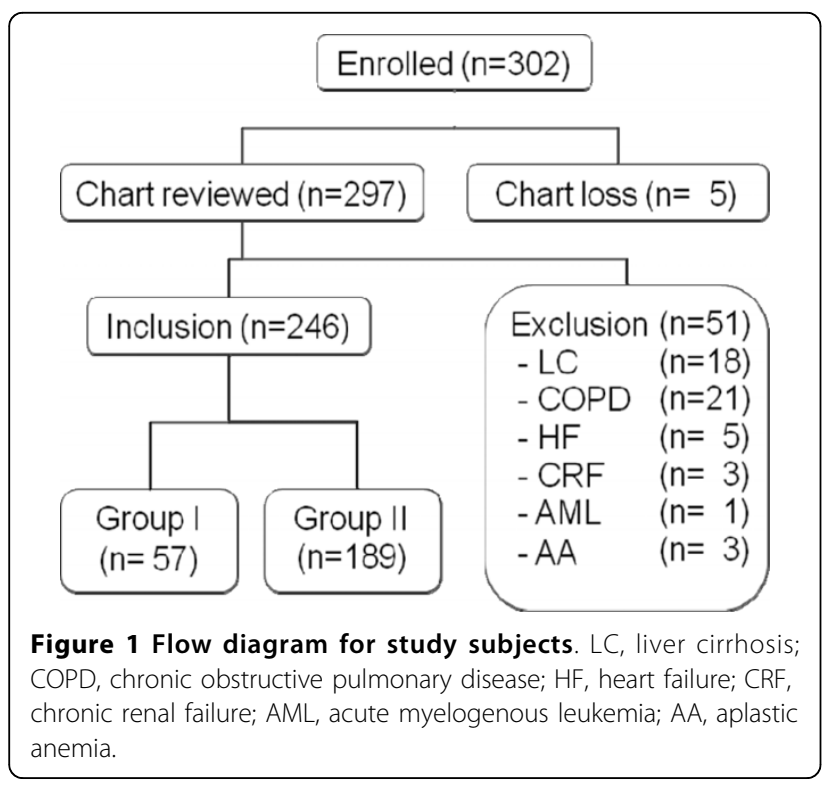

\section{Definitions}

Hypoalbuminemia was defined as a first serum albumin of less than $3.0 \mathrm{~g} / \mathrm{dL}$ on their initial visit [6,8]. Central nervous system (CNS) involvement was defined by the presence of CNS related symptoms such as confusion, seizure or coma. Involvement of respiratory system (RS) was defined by the presence of symptoms, signs and investigation results supporting evidence of pulmonary edema, interstitial pneumonia, pleural effusion or the need for mechanical ventilation. Cardiovascular system (CS) involvement was defined by the presence of symptoms, signs and investigation results supporting pericardial effusion, new onset of atrial fibrillation and ischemic heart disease. New onset of atrial fibrillation was defined as such when the symptoms improved with clinical recovery of scrub typhus. Gastrointestinal system (GS) involvement was defined by the presence of symptoms, signs and investigation results supporting gastric ulcer, pancreatitis, and upper gastrointestinal bleeding. Acute renal failure (ARF) was defined as such when at least $50 \%$ reduction was seen in glomerular filtration rate (GFR) using the abbreviated modified diet in renal disease (MDRD) equation, namely: GFR $(\mathrm{mL} / \mathrm{min} / 1.73$ $\left.\mathrm{m}^{2}\right)=186 \mathrm{P}_{\mathrm{cr}}^{-1.154} \times$ age $^{-0.203} \times(1.212$ if black $) \times(0.742$ if female) [14]. An "ST interval" was defined as an interval from the day when clinical symptoms began (prior to the admission) and to the day when appropriate treatment with antibiotics (Doxycycline or azithromycin) begun. An "AT interval" was defined as an interval from the day of admission to the day when appropriate treatment started.

\section{Costs}

All costs were expressed in US dollars. The currency rate was 1,300 Korean Won per 1 United States Dollar. We estimated only the direct medical cost, which included treatment (oral and/or intravenous administration), examination (laboratory and imaging studies), surgical and nonsurgical procedures, physical therapies, and room and board.

\section{Statistical analysis}

The results were analyzed using SPSS v 15.0 (SPSS, Inc., Chicago, IL, USA). Categorical variables were compared by Fisher's exact test or chi-square test and continuous variables were compared by the ANCOVA. All tests of significance were two-tailed; $\mathrm{p}$ values $\leq 0.05$ were considered to be significant.

\section{Results}

Since the age in Group I was significantly older than Group II $(p<0.001)$, we adjusted all variables for age to avoid biased outcome due to this demographic difference. Meanwhile, there were no statistical differences in 
gender and underlying diseases such as diabetes mellitus, hypertension, chronic hepatitis B and chronic hepatitis C.

As it might be expected from the symptoms and signs, hypoalbuminemic patients had much higher incidence of dyspnea $(p<0.001)$ compared to Group II, but the presence of typical signs of scrub typhus such as skin rash, eschar formation and lymphadenopathy and other vague complaints were not significant (Table $1)$. According to the rate of organ dysfunction, the rate of CNS $(p=0.006)$, RS $(p<0.013)$, GS $(p=0.012)$, and non-oliguric ARF $(p=0.001)$ were higher in Group I than in those of Group II, while the incidence of CS ( $p=0.299)$ differed not significantly between the two groups (Table 2).

A summary of initial laboratory findings for groups I and II is shown in Table 3. The serum albumin levels on admission in Group I and Group II were $2.69 \pm 0.22$ and $3.56 \pm 0.35 \mathrm{~g} / \mathrm{dL}$, respectively. In Group I, leukocyte count $(p=0.007)$, AST $(p=0.024)$ and total bilirubin $(p$ $<0.001)$, and BUN were higher, and platelet count was significantly lower $(p<0.001)$ compared to Group II. On the other hand, there were no statistical differences in hematocrit, hemoglobin, ALT, BUN, $\mathrm{Na}^{+}$, and $\mathrm{K}^{+}$ between the two groups. The GFR $(p=0.050)$ was

Table 1 Demographic and Clinical Characteristics of 246 Study Patients

\begin{tabular}{|c|c|c|c|}
\hline Characteristics & Group I & Group II & $P^{+}$ \\
\hline Age, mean $\pm S D$, years & $71.4 \pm 10.6$ & $60.7 \pm 12.2$ & $<0.001$ \\
\hline \multicolumn{4}{|l|}{ Sex } \\
\hline Male & $22(38.6)$ & $62(32.8)$ & 0.311 \\
\hline Female & $35(61.4)$ & $127(67.2)$ & \\
\hline \multicolumn{4}{|l|}{ Underlying disease } \\
\hline Diabetes Mellitus & $2(3.5)$ & $1(0.5)$ & 0.660 \\
\hline Hypertension & $16(28.1)$ & $45(23.8)$ & 0.734 \\
\hline Chronic hepatitis B & $0(0.0)$ & $5(2.6)$ & 0.997 \\
\hline Chronic hepatitis C & $2(3.5)$ & $1(0.5)$ & 0.288 \\
\hline \multicolumn{4}{|l|}{ Clinical manifestations } \\
\hline Chills & $47(82.5)$ & $166(87.8)$ & 0.658 \\
\hline Sore throat & $9(15.8)$ & $30(15.9)$ & 0.453 \\
\hline Headache & $35(61.4)$ & $124(65.6)$ & 0.566 \\
\hline Myalgia & $38(66.7)$ & $117(61.9)$ & 0.546 \\
\hline Arthralgia & $0(0.0)$ & $11(5.8)$ & 0.997 \\
\hline Cough & $20(35.1)$ & $66(34.9)$ & 0.600 \\
\hline Dyspnea & $26(45.6)$ & $38(20.1)$ & 0.012 \\
\hline Nausea/vomiting & $24(42.1)$ & $71(37.6)$ & 0.297 \\
\hline Abdominal pain & $24(42.1)$ & $55(29.1)$ & 0.103 \\
\hline Skin rash & $38(66.7)$ & $123(65.1)$ & 0.639 \\
\hline Eschar & $42(73.7)$ & $131(69.3)$ & 0.191 \\
\hline Lymphadenopathy & $3(5.3)$ & $20(10.6)$ & 0.434 \\
\hline Conjunctivitis & $5(8.8)$ & $24(12.7)$ & 0.759 \\
\hline
\end{tabular}

${ }^{\dagger}$ Adjusted for age; SD, standard deviation.
Table 2 Complications of the Patients in the study

\begin{tabular}{|c|c|c|c|}
\hline Complications & Group I & Group II & $p^{+}$ \\
\hline Central Nervous System & $17(29.8)$ & $14(7.4)$ & 0.006 \\
\hline Confusion & $14(24.6)$ & $10(5.3)$ & 0.021 \\
\hline Seizure & $2(3.5)$ & $1(0.5)$ & 0.191 \\
\hline Coma & $1(3.5)$ & $3(1.6)$ & 0.814 \\
\hline Respiratory System & $26(45.6)$ & $38(20.1)$ & 0.013 \\
\hline Pulmonary edema & $9(15.8)$ & $6(3.2)$ & 0.002 \\
\hline Pleural effusion & $13(22.8)$ & $21(11.1)$ & 0.162 \\
\hline Pneumonia & $1(1.8)$ & $9(4.8)$ & 0.044 \\
\hline Ventilator use & $3(5.3)$ & $2(1.1)$ & 0.141 \\
\hline Cardiovascular System & $9(15.8)$ & $10(5.3)$ & 0.299 \\
\hline Pericardial effusion & $1(1.8)$ & $1(0.5)$ & 0.456 \\
\hline Arrhythmia* & $7(12.3)$ & $5(2.6)$ & 0.215 \\
\hline Ischemic heart disease & $1(1.8)$ & $4(2.1)$ & 0.561 \\
\hline Gastrointestinal System & $10(17.5)$ & $10(5.3)$ & 0.012 \\
\hline Peptic ulcer & $6(10.5)$ & $7(3.7)$ & 0.226 \\
\hline Pancreatitis & $1(1.8)$ & $0(0.0)$ & 0.995 \\
\hline Upper gastrointestinal bleeding & $2(3.5)$ & $0(0.0)$ & 0.995 \\
\hline Cholecystitis & $0(0.0)$ & $2(1.1)$ & 0.997 \\
\hline Appendicitis & $1(1.8)$ & $1(0.5)$ & 0.168 \\
\hline Kidney & $27(47.4)$ & $27(14.3)$ & $<0.001$ \\
\hline Non-oliguric ARF & $23(40.4)$ & $21(11.1)$ & 0.001 \\
\hline Oliguric ARF & $4(7.0)$ & $6(3.2)$ & 0.561 \\
\hline
\end{tabular}

${ }^{+}$Adjusted for age; ARF, acute renal failure.

*Arrhythmia; new onset of atrial fibrillation.

marginally lower in Group I than in Group II. The ST $(p=0.406)$ and $\operatorname{AT}(p=0.487)$ interval was not significantly different between the two groups. Although the patients of Group I had higher APACHE II score $(p<$ $0.001)$, medical costs $(p<0.001)$ and longer hospital stay $(p<0.001)$ than the Group II, there was no difference in mortality $(p=0.796)$ (Table 4,5$)$.

Table 3 Admission Laboratory Findings of the study Patients*

\begin{tabular}{lccc}
\hline & Group I & Group II & $\boldsymbol{P}^{+}$ \\
\hline WBC $\left(10^{3} / \mathrm{mL}\right)$ & $10.79 \pm 4.04$ & $8.57 \pm 4.10$ & 0.007 \\
Hematocrit $(\%)$ & $30.87 \pm 10.98$ & $31.86 \pm 12.03$ & 0.869 \\
Hemoglobin $(\mathrm{g} / \mathrm{dL})$ & $11.63 \pm 1.92$ & $12.38 \pm 1.60$ & 0.067 \\
Platelet $\left(10^{3} / \mathrm{mm}^{3}\right)$ & $115.11 \pm 69.07$ & $161.28 \pm 90.92$ & $<0.001$ \\
AST $(\mathrm{IU} / \mathrm{L})$ & $121.60 \pm 85.95$ & $106.87 \pm 99.44$ & 0.024 \\
ALT $(\mathrm{IU} / \mathrm{L})$ & $107.35 \pm 168.92$ & $97.15 \pm 103.94$ & 0.084 \\
T-bili $(\mathrm{mg} / \mathrm{dL})$ & $1.417 \pm 1.33$ & $0.84 \pm 0.59$ & $<0.001$ \\
Albumin $(\mathrm{g} / \mathrm{dL})$ & $2.69 \pm 0.22$ & $3.56 \pm 0.35$ & $<0.001$ \\
BUN $(\mathrm{mg} / \mathrm{dL})$ & $32.94 \pm 17.05$ & $19.50 \pm 14.11$ & $<0.001$ \\
GFR $(\mathrm{mg} / \mathrm{dL})$ & $61.64 \pm 37.37$ & $79.51 \pm 33.16$ & 0.050 \\
$\mathrm{Na}{ }^{+}(\mathrm{mmol} / \mathrm{L})$ & $134.18 \pm 6.11$ & $135.19 \pm 11.14$ & 0.573 \\
$\mathrm{~K}^{+}(\mathrm{mmol} / \mathrm{L})$ & $3.70 \pm 0.70$ & $3.88 \pm 0.71$ & 0.109 \\
\hline
\end{tabular}

* Values are the mean \pm standard deviation; ${ }^{\dagger}$ Adjusted for age; WBC, white blood cell; AST, aspartate aminotransferase; ALT, alanine aminotransferase; BUN, blood urea nitrogen; GFR, glomerular filtration rate; $\mathrm{Na}^{+}$, sodium; $\mathrm{K}^{+}$, potassium. 
Table 4 The Comparison of ST, AT interval, APACHE II score, Intensive Care Unit Admission, Septic Shock, and Mortality of the Patients*

\begin{tabular}{|c|c|c|c|}
\hline & Group I & Group II & $P^{\dagger}$ \\
\hline ST interval, mean \pm SD & $6.84 \pm 4.14$ & $7.44 \pm 4.12$ & 0.406 \\
\hline AT interval, mean \pm SD & $0.98 \pm 1.89$ & $0.87 \pm 2.34$ & 0.487 \\
\hline APACHE II Score, mean \pm SD & $11.37 \pm 5.1$ & $6.94 \pm 4.2$ & $<0.001$ \\
\hline Intensive Care Unit Admission & $10(17.5 \%)$ & $13(6.9 \%)$ & 0.162 \\
\hline Septic Shock & $11(19.3 \%)$ & $15(7.9 \%)$ & 0.134 \\
\hline Mortality & $3(5.3 \%)$ & $6(3.2 \%)$ & 0.796 \\
\hline
\end{tabular}

* ST interval = from the day when clinical symptoms began and to the day when appropriate treatment with antibiotics began; AT interval = from the day of admission to the day when appropriate treatment started.

${ }^{+}$Adjusted for age; APACHE II, Acute physiology and chronic health evaluation; SD, standard deviation

\section{Discussion and Conclusion}

In this study, scrub typhus patients with hypoalbuminemia had a higher APACHE II score, longer hospital stay, and higher medical cost compared to the patients without hypoalbuminemia. And in the hypoalbuminemic group, thrombocytopenia and leukocytosis, which were known to be clinical parameters representing disease severity [15], were more severe. In addition, the hypoalbuminemic group showed a higher rate of complication in CNS, RS, GS, and non-oliguric ARF.

Generally, the mechanism of hypoalbuminemia in acute infectious disease is known to be related with poor oral intake of protein, decreased synthesis of protein from the liver, increased catabolism of protein, and increased metabolism of albumin due to the vascular leakage of serum protein due to increased vascular permeability [16]. A major and serious pathologic change of scrub typhus is a focal or disseminated vasculitis due to the destruction of endothelial cell lining of the small vessels, which is manifested as perivascular infiltration of leukocytes [17-19], an increased vascular permeability with extravascular protein loss, and consequently the hypoalbuminemia.

The widespread vasculitis or perivasuculitis in scrub typhus may involve the lung, cardiovascular system, brain, kidney, gastrointestinal tract, liver, and lymph nodes [4,17-21]. Song et al [8] showed that interstitial pneumonia, pleural effusion, and pulmonary edema were more frequently reported in scrub typhus patients with hypoalbuminemia than in patients without hypoalbuminemia. In this study, dyspnea and pulmonary edema occurred more frequently in the group with hypoalbuminemia than in the patients without hypoalbuminemia. This finding concurred with that of Song et al [8].

Previous reports showed that septic shock [22] and hepatic dysfunction [23] were frequently reported in patients with scrub typhus with hypoalbuminemia. They suggested that these complications were closely associated with the disease severity of scrub typhus. In this study, the level of AST and total bilirubin were higher in the hypoalbuminemia group than in the group without hypoalbuminemia.

Acute renal failure can occur due to acute tubular necrosis caused by direct invasion of Oriential tsutsugamushi [24]. Renal complications may prolong its morbidity and even lead to death. In this study, renal failure was more frequently reported in the group with hypoalbuminema. We suggest that the hypoalbuminemia has close association with acute renal failure. The absolute WBC counts varied but had a significantly higher mean value in the hypoalbuminemia group. This suggests that patients with hypoalbuminemia might have been more seriously ill.

Though hypoalbuminemia is a powerful predictor of mortality in patients with various illnesses [25-27], this study did not show significantly higher mortality in patients with hypoalbuminemia than the nonhypoalbuminemic patients. Incidence of severe scrub typhus is actually low, and an active treatment with antibiotics will suffice for managing the case. Therefore, the hypoalbuminemia itself may be insignificant in predicting poor clinical courses in scrub typhus. This suggestion can be supported by lower APACHE II score in hypoalbuminemic scrub typhus patients compared to other severe septic conditions.

The length of hospital stay in the hypoalbuminemic group was significantly longer than that of group without hypoalbuminemia, raising the cost of their hospital stay significantly higher for more tests and treatments. It is suggested that scrub typhus patients with hypoalbuminemia had more complications than the patients without hypoalbuminemia, although their mortality rate remained low.

Our study has some limitations. Firstly, our study is retrospective, which has obvious limitations. Secondly, we classified the level of albumin into two groups. Therefore, the clinical impact of hypoalbuminemia on patients with scrub typhus could not be evaluated. Thirdly, outcomes of albumin administration had not been evaluated.

Table 5 Comparison of Length of hospital Stay, and the Cost Between the Two Groups*

\begin{tabular}{|c|c|c|c|}
\hline & Group I & Group II & $P$ \\
\hline Duration of hospital Stay, mean \pm SD & $11.46 \pm 8.9$ & $5.93 \pm 5.9$ & $<0.001$ \\
\hline Cost (USD) & $2357.83 \pm 2284.15$ & $1135.97 \pm 1668.62$ & $<0.001$ \\
\hline
\end{tabular}

${ }^{\dagger}$ Adjusted for age; SD, standard deviation; 1 USD (united states dollar); 1,300 Won (Korean Currency). 
As the annual incidence of scrub typhus in Korea has been increasing steadily with its reported cases sharply rose to 6057 in 2008 [28], the financial burden of caring for scrub typhus is becoming an emerging problem. This study was done in search of more effective measures for improving patient care in scrub typhus. The hypoalbuminemia as a criterion is an important marker on the clinical outcome of patients with scrub typhus.

\section{Author details \\ 'Department of Internal Medicine, Chonbuk National University Medical School and Research Institute of Clinical Medicine, Geumam-dong, Jeonju, 561180, Republic of Korea. ${ }^{2}$ Chonbuk National University Medical School, Geumam-dong, Jeonju, 561180, Republic of Korea. ${ }^{3}$ Department of Internal Medicine, Chonbuk National University Medical School, Geumam-dong, Jeonju, 561180, Republic of Korea. ${ }^{4}$ Preventive Medicine, Chonbuk National University Medical School and Research Institute of Clinical Medicine, Geumam-dong, Jeonju, 561180, Republic of Korea.}

\section{Authors' contributions}

CSL contributed to the concept, design, conduct and analysis of the study and also wrote the manuscript. ISM contributed to the study design, conduct and also wrote the manuscript. JHH contributed to the study concept, design and interpretation and provided a critical review of the manuscript. KSK contributed to the design, implementation of the study, provided an oversight of data collection and statistical analysis. HBL contributed to the design and interpretation of the results and a critical review of the manuscript. All authors read and approved the final manuscript.

\section{Competing interests}

The authors declare that they have no competing interests.

Received: 5 March 2010 Accepted: 21 July 2010 Published: 21 July 2010

\section{References}

1. Saah A: Orientia tsutsugamushi (scrub typhus). Principles and practice of infectious disease Philadelphia: Churchill LivingstoneMandell GL, Bennett JE, Dolin R, 5 2000, 2:2056-2057.

2. Watt G, Parola P: Scrub typhus and tropical rickettsioses. Curr Opin Infect Dis 2003, 16:429-436.

3. Tsay RW, Chang FY: Serious complications in scrub typhus. J Microbiol Immunol Infect 1998, 31:240-244.

4. Chi WC, Huang JJ, Sung JM, Lan RR, Ko WC, Chen FF: Scrub typhus associated with multiorgan failure: A case report. Scand J Infect Dis 1997, 29:634-635.

5. Hsu GJ, Young T, Peng MY, Chang FY, Chou MY, Sheu LF: Acute renal failure associated with scrub typhus: Report of a case. J Formos Med Assoc 1993, 92:475-477.

6. Carratala J, Roson B, Fernandez-Sabe N, Shaw E, del Rio O, Rivera A: Factors associated with complications and mortality in adult patients hospitalized for infectious cellulitis. Eur J Clin Microbiol Infect Dis 2003, 22:151-157.

7. Jang KM, Kang MH, Yang YS, Hwang HG, Lee KP, Lee JS: The twenty cases of serologically confirmed tsutsugamushi disease. J Korean Med Assoc 1987, 30:638-646.

8. Song SW, Kim KT, Ku YM, Park SH, Kim YS, Lee DG, Yoon SA, Kim YO: Clinical role of interstitial pneumonia in patients with scrub typhus: A possible marker of disease severity. J Korean Med Sci 2004, 19:668-673.

9. Kim DM, Yun NR, Yang TY, Lee JH, Yang JT, Shim SK, Choi EN, Park MY, Lee SH: Usefulness of nested PCR for the diagnosis of scrub typhus in clinical practice: A prospective study. Am J Trop Med Hyg 2006, 75:542-545.

10. Kim IS, Seong SY, Woo SG, Choi MS, Kang JS, Chang WH: Rapid diagnosis of scrub typhus by a passive hemagglutination assay using recombinant 56-kilodalton polypeptides. J Clin Microbiol 1993, 31:2057-2060.
11. Song HJ, Seong SY, Huh MS, Park SG, Jang WJ, Kee SH, Kim KH, Kim SC, Choi MS, Kim IS, Chang WH: Molecular and serologic survey of orientia tsutsugamushi infection among field rodents in southern cholla province, korea. Am J Trop Med Hyg 1998, 58:513-518.

12. Kim YS, Yun HJ, Shim SK, Koo SH, Kim SY, Kim S: A comparative trial of a single dose of azithromycin versus doxycycline for the treatment of mild scrub typhus. Clin Infect Dis 2004, 39:1329-1335.

13. Chang WH, Kang JS, Lee WK, Choi MS, Lee JH: Serological classification by monoclonal antibodies of rickettsia tsutsugamushi isolated in korea. $J$ Clin Microbiol 1990, 28:685-688.

14. Manjunath G, Sarnak MJ, Levey AS: Prediction equations to estimate glomerular filtration rate: An update. Curr Opin Nephrol Hypertens 2001, 10:785-792.

15. Wang CC, Liu SF, Liu JW, Chung YH, Su MC, Lin MC: Acute respiratory distress syndrome in scrub typhus. Am J Trop Med Hyg 2007, 76:1148-1152.

16. Kim YO, Yoon SA, Ku YM, Yang CW, Kim YS, Kim SY, Choi EJ, Chang YS, Bang BK: Serum albumin level correlates with disease severity in patients with hemorrhagic fever with renal syndrome. J Korean Med Sci 2003, 18:696-700.

17. Strickman $D$, Smith $C D$, Corcoran $K D$, Ngampochjana M, Watcharapichat $P$, Phulsuksombati D, Tanskul P, Dasch GA, Kelly DJ: Pathology of rickettsia tsutsugamushi infection in bandicota savilei, a natural host in thailand. Am J Trop Med Hyg 1994, 51:416-423.

18. Kim SJ, Chung IK, Chung IS, Song DH, Park SH, Kim HS, Lee MH: The clinical significance of upper gastrointestinal endoscopy in gastrointestinal vasculitis related to scrub typhus. Endoscopy 2000, 32:950-955.

19. Seong SY, Choi MS, Kim IS: Orientia tsutsugamushi infection: Overview and immune responses. Microbes Infect 2001, 3:11-21.

20. Hwang TS, Chu YC, Kim YB, Lim BU, Kang JS: Pathologic study of mice infected with rickettsia tsutsugamushi r19 strain. J Korean Med Sci 1993, 8:437-445.

21. Sirisanthana V, Puthanakit T, Sirisanthana T: Epidemiologic, clinical and laboratory features of scrub typhus in thirty thai children. Pediatr Infect Dis J 2003, 22:341-345.

22. Thap LC, Supanaranond W, Treeprasertsuk S, Kitvatanachai S, Chinprasatsak S, Phonrat B: Septic shock secondary to scrub typhus: Characteristics and complications. Southeast Asian I Trop Med Public Health 2002, 33:780-786.

23. Chanta C, Triratanapa K, Ratanasirichup P, Mahaprom W: Hepatic dysfunction in pediatric scrub typhus: Role of liver function test in diagnosis and marker of disease severity. J Med Assoc Thai 2007, 90:2366-2369,

24. Kim DM, Kang DW, Kim JO, Chung JH, Kim HL, Park CY, Lim SC: Acute renal failure due to acute tubular necrosis caused by direct invasion of Oriential tsutsugamushi. J Clin Microbiol 2008, 46:1548-1550.

25. Zisman DA, Kawut SM, Lederer DJ, Belperio JA, Lynch JP, Schwarz MI, Tayek JA, Reuben DB, Karlamangla AS: Serum albumin concentration and waiting list mortality in idiopathic interstitial pneumonia. Chest 2009, 135:929-935.

26. Boonpipattanapong T, Chewatanakornkul S: Preoperative carcinoembryonic antigen and albumin in predicting survival in patients with colon and rectal carcinomas. J Clin Gastroenterol 2006, 40:592-595.

27. Franch-Arcas $\mathrm{G}$ : The meaning of hypoalbuminaemia in clinical practice. Clin Nutr 2001, 20:265-269.

28. Korea Center for Disease Control and Prevention: Disease web statistics system. [http://stat.cdc.go.kr/], Accessed 11 July 2010.

\section{Pre-publication history}

The pre-publication history for this paper can be accessed here: http://www.biomedcentral.com/1471-2334/10/216/prepub

\section{doi:10.1186/1471-2334-10-216}

Cite this article as: Lee et al:: Clinical significance of hypoalbuminemia in outcome of patients with scrub typhus. BMC Infectious Diseases 2010 $10: 216$. 